\title{
LA TEORÍA LÓGICA DE FRANZ BOLZANO: UNA REACCIÓN CONTRA EL SUBJETIVISMO KANTIANO
}

\author{
Pilar CASTRILLO \\ Dpto. de Lógica, Historia y Filosofia de la Ciencia. \\ UNED
}

ABSTRACT: Objectivism may be taken as a landmark of Austrian philosophy. This tradition begins with Bernard Bolzano. Contrary to the Kantian picture of the working of the human mind as imposing its own structure on nature, Bolzano stresses the objectivity of our grasping the truth, thus liberating cognition from the subjective constraints. To know something means always to know propositions as such. Bolzano's theory states that there are propositions in themselves which are the bearers of truth values and of logical relations.

\section{Introducción}

A pesar de que su teoría del juicio es una de las partes más pobres de su filosofía y de que su interés por la lógica fue sólo superficial, a Kant le corresponde, sin embargo, el mérito de haber hecho de la formalidad el rasgo distintivo de esta disciplina. En el Prefacio de la segunda edición de la Crítica de la razón pura, tras su célebre afirmación de que la lógica no ha logrado avanzar un solo paso desde los tiempos de Aristóteles, "presentándose, según todas las apariencias como conclusa y perfecta», subraya Kant: «Las fronteras de la lógica se hallan delimitadas con absoluta precisión por la peculiaridad de tratarse de una ciencia a la que sólo le interesa la detallada exposición y la rigurosa demostración de las reglas formales de todo pensamiento" (KrV B VIII).

La doctrina de que la formalidad constituye la característica definitoria de la lógica alcanzó una gran popularidad en el siglo XIX, siendo muchos los autores que reconocieron sus orígenes kantianos. Y es que, aunque siempre se ha hecho más hincapié en la influencia de Leibniz en el proceso de constitución de la lógica actual, lo cierto es que todos cuantos tuvieron alguna intervención en el mismo, incluso aquellos que rechazaron los presupuestos de la filosofia kantiana y presentaron sus contribuciones como una alternativa a sus doctrinas, reconocieron el valor de las ideas de Kant como fuente de inspiración. Tal es el caso del 
matemático, filósofo y lógico checo Bernard Bolzano, que aunque fue uno de los más tempranos y radicales críticos de la obra de Kant, no dejó de hacerse eco de muchas de sus doctrinas, especialmente de la naturaleza formal de la lógica, que en su obra cobra especial relieve por constituir la base de su caracterización de los conceptos de verdad lógica y consecuencia lógica.

Nacido en Praga en 1781, año de la aparición de la primera edición de la Crítica de la razón pura, y desaparecido en el turbulento año de 1848 , Bernard Bolzano es uno de esos autores que ha permanecido sepultado bajo la pesada losa del olvido hasta tiempos bien recientes ${ }^{1}$, pese a ser muy probablemente, junto con Brentano, la figura más importante de la filosofía austríaca del siglo XIX. Como ha escrito Peter Simons: «El filósofo austríaco más importante ha sido también aquel al que la historia posterior ha tratado con más injusticia, al ser condenado a ver cómo sus descubrimientos eran hechos célebres por otros: su antipsicologismo y su platonismo respecto a los significados por Frege y Husserl, su rigor analítico por Cauchy y Weierstrass, sus conceptos semánticos por Tarski. Si su obra hubiera sido conocida antes, la lógica hubiera podido ahorrarse decenas de ańos en su desarrollo" (P. Simons, 1992, p. 6). Este aciago destino se explica por la confluencia de una serie de factores de muy distinta naturaleza. Lo primero que habría que destacar es el hecho de que Bolzano fue una de las muchas víctimas de la censura política que se practicaba en aquel momento dentro de los confines del imperio austro-húngaro, un hombre que desde que, en 1819, es apartado de la docencia que venía ejerciendo en la universidad de Praga, se vio obligado a llevar una existencia semi-clandestina, cosa que en modo alguno facilitó el conocimiento y la difusión de su obra. Por lo demás, en el caso de sus aportaciones a la lógica, otro factor que cabría reseñar como responsable de este olvido es la falta de interés que mostró por la construcción de sistemas deductivos. Es no ya posible sino sumamente probable que la ausencia de su obra de una maquinaria deductiva y el hecho de que su pensamiento en este aspecto se mantuviera al margen de la dirección en que soplaban los vientos de la modernidad hayan contribuido

${ }^{1}$ El primer autor de renombre que señala la importancia de la obra lógica de Bolzano fue Husserl, quien en sus Prolegómenos a la lógica pura se refiere a él como «uno de los mayores lógicos de todos los tiempos» (Husserl 1900, vol. I, p. 125). Pero esta obra no será objeto de atención hasta los estudios realizados, en los años 50, por el lógico $\mathrm{Y}$. Bar-Hillel, alumno en Israel de $\mathrm{H}$. Bergmann, autor él mismo de una monografía sobre Bolzano, y por J. Berg en los 60 . 
también, en no escasa medida, a que ésta no haya suscitado antes entre los historiadores de la lógica la atención que sin duda merece. Sin embargo, parece fuera de toda duda que el proceso de constitución de la lógica moderna no es obra únicamente de aquellos autores que, como es el caso de Boole o de Frege, se encargaron de diseñar sistemas formales, mostrando por vía de ejemplo que, en contra de lo que se había venido pensando durante tanto tiempo, la lógica podía ser estudiada completamente al margen de los procesos mentales. Es fruto también del quehacer de algunos pensadores que, si bien no mostraron especial interés por la construcción de cálculos lógicos particulares, no por ello dejaron de pergeñar importantes ideas sin las cuales la lógica actual no sería la que es. Tal es el caso de nuestro autor, y no sólo por haber introducido conceptos tan importantes en la lógica actual como los de validez, consecuencia y verdad formal, sino por la labor que realizó en defensa de un estatuto para la lógica autónomo e independiente de la psicología.

Como no podía menos de ocurrir en un buen seguidor de Leibniz y de Wolff, en el centro de las preocupaciones de Bolzano se encuentra la filosofía; sin embargo, la suerte corrida por Bolzano en la historiografía de la filosofía no puede decirse que haya sido mucho mejor. Si nos atenemos a las historias al uso, tal parece como si el siglo XIX hubiera sido únicamente el siglo de la filosofía alemana y de pensadores como Fichte, Hegel, Nietzsche o Marx. Sin embargo, por esa misma época, en algunas de las ciudades del imperio austro-húngaro se estaba gestando, gracias al trabajo de filósofos como Bolzano, Brentano, Husserl, Meinong o Twardowski, por no mencionar sino los más importantes, una filosofía con unas señas de identidad claramente definidas. Uno de los rasgos más destacados de esta tradición es la preocupación que prácticamente todos sus representantes mostraron por la claridad y exactitud en el uso de los conceptos. Bolzano fue un abanderado de esta convicción acerca del modo correcto de hacer filosofía, hasta el punto de hacerse acreedor al título de 'Meister des Verdeutlichens' o maestro clarificador que le otorgó uno de los primeros estudiosos de su obra (H. Bergmann 1909 , p. 53) ${ }^{2}$. Esto explica que se sintiese especialmente incómodo con lo que consideraba "la espantosa confusión" (die heillose Verwirrung) ${ }^{3}$ que los filosofemas de Kant habían ocasionado en Alemania y que

\footnotetext{
${ }^{2}$ Sobre los rasgos característicos de esta tradición austríaca y el lugar ocupado por Bolzano en ella, pueden verse R. Haller 1992 y K. Mulligan 1986.

${ }^{3} \mathrm{La}$ expresión aparece en una recomendación que le hace a su discípulo directo Robert Zimmermann, en la que le insta a trabajar para acabar con tal confusión (Cfr. Morscher 1973, p. 33).
} 
considerara tarea urgente acabar con ella, y explica igualmente que sus aportaciones más importantes en el campo de la lógica estriben, como trataremos de mostrar en las páginas que siguen, en la clarificación de nociones fundamentales, que si no logró definir con la precisión que hoy tienen, no fue por falta de perspicacia lógica sino únicamente por falta del aparato técnico necesario ${ }^{4}$.

\section{Rechazo del intuicionismo y el psicologismo}

En líneas generales puede afirmarse que el desacuerdo con las soluciones ofrecidas por Kant a los problemas relacionados con la naturaleza de las matemáticas y la búsqueda de una alternativa a las mismas constituyen una especie de hilo conductor en el desarrollo del pensamiento de Bolzano. Una de las fuentes más importantes de disensión entre ellos es la distinta concepción que uno y otro tienen del papel que debe desempeñar la lógica en las matemáticas, pues, mientras que para Kant éste es más bien secundario e incluso superfluo, dada la importancia que para él tiene la construcción intuitiva, Bolzano le conferirá, en cambio, un papel destacado a tono con su total desconfianza en la intuición como fuente de justificación de las matemáticas. Por una serie de razones que tienen que ver con el desarrollo logrado por esta materia, entre las que destaca especialmente el hecho de que las matemáticas de su tiempo trataran de objetos o conceptos que no eran susceptibles de una intuición al estilo kantiano, Bolzano no tardaría mucho, en efecto, en llegar a la conclusión de que ésta no puede constituir una base aceptable para las matemáticas y de que, en consecuencia, es preciso buscar un fundamento más seguro y general para las mismas 5 .

El cometido que se propone Bolzano y el núcleo en torno al cual gira su proyecto no es otro, en efecto, que el de ofrecer una explicación del conocimiento matemático, para él a priori y necesario, en la que la intuición no desempeñe papel alguno. Este proyecto chocaba frontalmente con la filosofía crítica kantiana por tratarse de una filosofía que entendía que en los juicios matemáticos,

4 "Si quisiera describir en una palabra la diferencia esencial entre mis ideas filosóficas ... y las de otros, habría de decir que reside en el hecho de que siempre he tenido como norma la preocupación por plantear todos mis pensamientos en un nivel más elevado de claridad y distinción que el que ha sido usual hasta ahora" (Bolzano, carta a Romang, de 1847), recogida en Berg 1962, p. 9.

${ }^{5}$ Bolzano cré́a que la intuición, aun la intuición pura, permanece siempre en el plano de lo finito y que no puede haber una intuición de las totalidades infinitas. Este tema del infinito ocupó siempre un papel central en la reflexión de Bolzano y a él dedicará la última y más conocida de sus obras, Las paradojas del infinito (1851). 
«aunque el predicado está vinculado necesariamente al sujeto, lo está en virtud de una intuición que ha de añadirse al concepto» ( $\mathrm{KrV}$ B17).

Bolzano venía ya mostrando su desacuerdo con el papel otorgado por Kant a la intuición matemática desde sus primeros escritos acerca de la naturaleza del conocimiento matemático. Así, en sus Beiträge zu einer begründeteren Darstellung der Mathematik (Contribuciones a una presentación de las matemáticas sobre mejores fundamentos), de 1810, podemos leer :

En lo que a mí respecta, reconoceré con toda franqueza que hasta ahora -al igual que me ocurre de hecho con tantas otras doctrinas de la filosofía críticahe sido incapaz de aceptar la corrección de las afirmaciones kantianas acerca de la intuición pura y de la construcción de conceptos a través de ella. Sigo creyendo que en el concepto de intuición pura (i.e., a priori) hay una contradicción interna; e incluso tengo más dificultades para convencerme a mí mismo de que es necesario construir el concepto de número en el tiempo y que, en consecuencia, la intuición del tiempo es parte fundamental de la aritmética ${ }^{6}$.

Y es que, aunque la presencia dominante del nombre de Kant en ésta como en otras de sus obras denota el gran respeto que este autor le merecía, el matemático checo estaba, sin embargo, lejos de suscribir los oscuros presupuestos en los que, en su opinión, se apoyaba la filosofía crítica kantiana. En concreto, se oponía a lo que constituye el núcleo central de la misma, a saber, la idea de que la intuición pura ha de fundamentar los juicios sintéticos a priori de la matemática, igual que la intuición empírica hace lo propio con los juicios a posteriori. Como habría de hacer Frege unos años después, el filósofo checo partía de la base de que habla que restringir al máximo el papel justificativo de la intuición en las matemáticas y evitar en la medida de lo posible la intrusión de elementos subjetivos en dicho dominio. Por otra parte, la fundamentación de la matemática, y más en concreto del análisis, que Bolzano perseguía, no estriba, como en el caso de Kant, en un intento de justificar una práctica dada con una teoría filosófica general, sino que consiste más bien en una reorganización rigurosa y siste-

${ }^{6}$ B. Bolzano, Beiträge..., cit. en L. Novy'1981, p. 107. Bolzano incluyó como Apéndice a este texto un ensayo titulado "Sobre la doctrina kantiana de la construcción de conceptos mediante las intuiciones", que constituye la primera crítica de la filosofia de la matemática kantiana. Como es bien sabido, Kant llama 'intuición pura' a «la forma pura de la sensibilidad" y dice de ella que se halla en la mente a priori.(KrV B34). 
mática de sus principios y en una elucidación precisa de sus métodos. Bolzano es, en efecto, uno de los primeros impulsores de un nuevo tipo de rigor argumentativo en las matemáticas y de la idea de que la definición y demostración analíticas, sin recurso a elementos extraños a la naturaleza de esta disciplina, son lo único que puede legitimar la verdad matemática. Este modo de enfocar las cosas, junto con las aportaciones concretas a las que llegó en la práctica por esta vía, hacen de él el iniciador del proceso de la llamada aritmetización del análisis, proceso que acabaría por desembocar en la creación de una teoría matemática unificadora. El programa bolzaniano de una Grössenlehre no es, efectivamente, sino el de una teoría comprensiva y unitaria de la matemática que obedece a la idea de que la totalidad de ésta es susceptible de una ordenación jerárquica y sistemática a partir de un conjunto relativamente pequeño de nociones fundamentales. Este programa, que desembocaría en la creación de la teoría de conjuntos, fue completado por figuras de la talla de Cauchy, Weierstrass, Dedekind y Cantor, muchas de las cuales por cierto no dejarían de evocar su memoria y de mostrar un reconocimiento explícito hacia su obra ${ }^{7}$.

Pero si Bolzano no está de acuerdo con Kant en lo que se refiere al papel otorgado por éste a la intuición en la explicación y justificación de las matemáticas, tampoco lo está en lo que concierne a su modo de entender las relaciones de la lógica con aquella ciencia. Aunque no fue un logicista en el sentido estricto del término, por cuanto que no propugnó la reducción de la matemática, o de ciertas partes de la misma, a la lógica, Bolzano no dejó de creer, no obstante, que la lógica es claramente presupuesta por las matemáticas. El filósofo bohemio hace suya, en efecto, la tesis leibniziana de la necesidad de dotar a las matemáticas de una fundamentación lógica, pudiendo verse su sistema como el primer intento verdaderamente moderno de acercamiento de las matemáticas a la lógica. La lógica se convierte así a sus ojos en una herramienta imprescindible para la investigación de los fundamentos de la matemática. Pero ¿qué lógica era la adecuada para desempeñar este papel? ¿Acaso lo era la mezcla de epistemología y psicología que pasaba por tal en su época ? Bolzano creía que no, y esta idea le llevaría a la convicción de que la primera tarea que había que abordar era la de someter a esta disciplina a una completa renovación.

${ }^{7}$ Cantor, por ejemplo, lo considera uno de los filósofos más agudos del siglo XIX, especialmente por haber mostrado en sus Paradojas del infinito que las contradicciones que los escépticos y peripatéticos han pretendido hallar en el infinito en realidad no existen. 
Aunque el estado de esta lógica no era en realidad sino una consecuencia de un largo proceso de descomposición que había tenido su inicio bastante tiempo atrás, Bolzano hacía a Kant y a su aparato conceptual responsables del mismo, y no le faltaban razones para ello. $Y$ es que, a pesar de que, como hemos señalado al principio, a Kant no se le puede negar el mérito de haber afirmado frente a los neo-leibnizianos de su tiempo la formalidad de la lógica, lo cierto es que esta declaración no se tradujo en un proyecto real ni en unas directrices que llevar a la práctica, sino que se redujo a una doctrina que de hecho quedaría eclipsada por otras presuposiciones del sistema filosófico kantiano en cierto modo incompatibles con ella. Pero conviene ir por partes. Lo primero que hay que empezar por reconocer es que es innegable que, al menos a partir del momento en que escribe la Crítica, Kant mantuvo explícitamente la idea de que la lógica pura, en tanto que distinta de las lógicas 'aplicada', 'especial' o 'transcendental', es una disciplina formal, esto es, una disciplina que

hace abstracción de todo contenido del conocimiento, i.e., de toda relación de éste con los objetos, y considera únicamente la forma lógica de la relación de los conocimientos unos con otros, i.e., la forma del pensamiento en general (KrV, A55/B79; Cfr. A56/B80; A70/B95; A131/B170).

Decir que la lógica es formal en este sentido equivale a decir que es insensible a los contenidos de los conceptos y juicios y que atiende únicamente a las formas. Es precisamente por esta razón por lo que, a juicio de Kant y contrariamente a lo mantenido por Frege, la lógica no puede ampliar nuestro conocimiento de la realidad, de los objetos ( $\mathrm{KrV}$ A60/B85). Ahora bien, la formalidad no era, para Kant, un rasgo definitorio aparte, sino una mera consecuencia de la generalidad de la lógica. Esto se ve con toda claridad en la discusión acerca de la naturaleza de esta disciplina incluida en la Lógica de Jäsche, en donde podemos leer: ${ }^{8}$

[1] Si ahora dejamos de lado todo conocimiento proveniente de los objetos y nos limitamos a reflexionar únicamente sobre el uso de muestro entendimiento, descubrimos aquellas de sus reglas que son necesarias sin cualificación, para

${ }^{8}$ Esta Lógica kantiana es una recopilación realizada por Gottlob Benjamin Jäsche a partir de apuntes de alumnos y notas del propio Kant. Para algunas de las cuestiones que se plantean acerca de su autenticidad y algunos ejemplos de divergencias entre el texto y las notas de Kant, véase T. Boswell 1988. 
todo propósito y sin referencia a ningún objeto particular del pensamiento, porque sin ellas no pensaríamos en modo alguno. [2] De este modo, podemos conocer tales reglas a priori, i.e con independencia de toda experiencia, porque meramente contienen las condiciones para el uso del entendimiento en general, sin distinción entre sus objetos, sea este uso puro o empírico. [3] De esto se deduce al mismo tiempo que las reglas universales y necesarias del pensamiento en general sólo pueden ocuparse de su forma y en modo alguno de su materia.[4] En consecuencia, la ciencia que contiene esas reglas universales y necesarias es meramente una ciencia de la forma de nuestro conocer a través del entendimiento, o del pensamiento (Kant 1800, p. 12).

En pocas palabras, para Kant la lógica es general en el sentido de que ofrece las normas constitutivas del pensamiento en cuanto tal, y de esta generalidad se siguen la aprioricidad y la formalidad de la misma. No es, pues, sino una propedeútica para el uso del entendimiento en cuanto tal, siendo ésta la razón de que no sea un 'organon' (nombre con el que se denomina al viejo ars inveniendi), sino «un canon del entendimiento y la razón, pero sólo respecto de lo que es formal en su uso, sea cual sea el contenido" (KrV A53/B77; Cf. Kant 1800, pp. 1314). Esto es, la lógica contiene, al decir de Kant, aquellas reglas sin las cuales no es posible ningún uso del entendimiento.

En ninguno de estos textos programáticos cabe rastrear en principio indicio alguno de psicologismo o defensa alguna de la idea de que es necesario fundamentar la lógica en la psicología. De hecho, Kant no parece haber creído que la necesidad de la lógica pueda fundamentarse en una ciencia empírica como es la psicología e incluso llega a afirmar explícitamente que introducir principios psicológicos en lógica "sería tan absurdo como derivar la moralidad de la vida" (Kant 1800 , p. 14). No obstante esto, no es menos cierto que la filosofía kantiana, y más en concreto su transcendentalismo, encargado de atribuir al entendimiento el poder constituyente de las verdades, partía de presupuestos que contenían en germen ideas proclives a una concepción psicologista y subjetivista de la lógica. Así lo pusieron de manifiesto las interpretaciones realizadas por algunos de sus epígonos, especialmente Beneke y Fries, que con toda razón se han hecho acreedores a la paternidad de esta doctrina, doctrina a la que, como consecuencia de todo esto, el nombre de Kant ha permanecido asociado desde entonces.

La tesis según la cual los principios de la lógica no son sino descripciones de los estados y procesos mentales que ocurren cuando razonamos, conocida con el 
nombre de 'psicologismo', no es sino una de las propuestas de explicación de la naturaleza de la lógica. Semejante propuesta encontró un suelo especialmente abonado en la hostilidad hacia las entidades abstractas propia del empirismo inglés y en su convicción de que todo conocimiento es conocimiento empírico. De hecho, uno de sus máximos representantes de esta doctrina es J. Stuart Mill, que, como es sabido, pertenece a esta corriente de pensamiento. Pero el psicologismo rebasa en mucho el ámbito del empirismo y su defensa no es siquiera un indicio inequívoco de asunción de ideas empiristas, por cuanto que ciertas formas de racionalismo trataron de basar las verdades necesarias en las estructuras esenciales o transcendentales de la mente. En este sentido, el psicologismo propiamente dicho suele asociarse con un movimiento filosófico que floreció en Alemania especialmente en la segunda mitad del siglo XIX y cuyas figuras más representativas fueron Wundt, Sigwart, Erdmann o Lipps ${ }^{9}$. Aunque así formulada hoy nos parece una teoría totalmente insostenible, lo cierto es que la doctrina de que la lógica tiene que ver con el estudio de los procesos del pensamiento fue bastante difícil de erradicar; de hecho su erradicación completa no se lograría sino a con las duras críticas de Frege y de Husserl de finales de dicho siglo. Pero estas críticas no fueron sino la culminación de un largo proceso que tuvo su inicio en la labor realizada por nuestro autor, quien claramente descontento con la manera subjetivista de entender la lógica, será el primero, en los tiempos modernos, en asumir con claridad el proyecto de asentar esta disciplina sobre bases más sólidas y objetivas. La estrategia de la que se valió para ello no fue otra que la de contraponer a la doctrina psicologista la tesis, que analizaremos a continuación, de que la lógica se ocupa de entidades abstractas y de las conexiones objetivas entre tales entidades ${ }^{10}$.

\section{La objetividad de las proposiciones}

Uno de los principales motivos alegados para ello por quienes tachan a Kant de psicologista es que incurrió permanentemente en una confusión entre los elementos subjetivo y objetivo de las representaciones (Vorstellungen), haciendo especial hincapié en el primero, esto es, entendiendo por representaciones estados

${ }^{9}$ Para un análisis sociológico de esta corriente de pensamiento y de los muchos filósofos que formaron parte de la misma o que, con o sin fundamento, fueron tachados de psicologistas, véase. M. Kusch 1995.

${ }^{10} \mathrm{La}$ tesis de que Bolzano fue el primero en defender una concepción claramente antipsicologista de la lógica, pese a que no dejó de hacer algunas manifestaciones explícitas que pueden interpretarse en sentido contrario, es defendida convincentemente en R. George 1997. 
mentales o "determinaciones del alma" — como él las llama— allí donde lo que hubiera procedido considerar es la representación objetiva. Consciente de los problemas que se derivan de esta ambigüedad, lo primero que va a hacer Bolzano es establecer una clara distinción entre las representaciones subjetivas y las representaciones objetivas o 'representaciones en si', y restringir el análisis lógico al ámbito de estas últimas y al de las proposiciones en sí, de las que no son sino los elementos constitutivos.

Las ideas lógicas de Bolzano se hallan contenidas fundamentalmente en la voluminosa obra en cuatro volúmenes publicada en 1837 con el título Wissenschaftslehre ${ }^{11}$. En el apartado 15 de esta obra, cuyo título completo es Teoría de la Ciencia o Intento de una exposición detallada y, en lo fundamental, novedosa de la lógica con atención constante a otros autores, Bolzano expone el plan de la misma, señalando los contenidos que, en su opinión, debe abarcar la lógica y que son los siguientes:

1. Teoría fundamental. Demostración de que existen las verdades en sí y de que el ser humano tiene la capacidad de conocerlas.

2. Teoría Elemental. Teoría de las representaciones en sí, de las proposiciones en sí y de la verdad e inferencia en sí.

3. Teoría del conocimiento. De las condiciones a las que se halla sometido el conocimiento de la verdad.

4. Heurística o de las reglas que han de observarse cuando de hallar la verdad se trata.

5. Teoría de la ciencia en sentido estricto o de las reglas que han de seguirse en la división del ámbito de la verdad en las ciencias particulares y en la exposición de las mismas en libros de texto.

Como vemos, el concepto bolzaniano de lógica es mucho más amplio de lo que hoy entendemos por tal: incluye no sólo una teoría de la inferencia válida sino toda una teoría general de la ciencia, atendiendo además no ya sólo a los aspectos epistemológicos sino también a los de descubrimiento e incluso a los de

${ }^{11}$ De estos cuatro volúmenes, sólo los dos primeros se ocupan de lógica en el sentido actual del término. Nos referiremos a esta obra mediante las siglas $W L$, seguidas de un número que se referirá siempre al apartado correspondiente de la misma. 
exposición. Desde el punto de vista de la lógica tal y como hoy la entendemos, lo más interesante de esta obra son las secciones que componen la Teoria elemental. La noción central de la lógica que en ella se expone es la de 'proposición en sí (Satz an sich) o proposición objetiva, que constituye el arma de la que va a valerse su autor para establecer la objetividad e independencia y autonomía de esta materia. La postulación de proposiciones objetivas o estados de cosas (o ambas cosas a la vez) como medio para salvaguardar la objetividad de la lógica y garantizar su independencia tanto respecto de la epistemología como de la psicología no es privativa de Bolzano, sino que es un recurso del que echaron mano otros representantes de la filosofía austríaca como Brentano, en su primera etapa, Marty o Meinong, pudiendo decirse que es un rasgo característico de las teorías de la lógica desarrolladas dentro de esta tradición ${ }^{12}$.

Entiende Bolzano por 'proposición en sí' - un claro precedente de la noción fregeana de 'pensamiento' - "cualquier afirmación de que algo es o no es el caso, al margen de si alguien lo ha puesto en palabras y al margen incluso de si ha sido pensado" (WL 19). Es decir, Bolzano diferencia claramente las proposiciones de sus correlatos psicológicos (pensamientos o juicios), de un lado, y de sus correlatos lingüísticos (oraciones), de otro. "Al hablar de una proposición enunciada, distingo claramente la proposición misma de su enunciación; de igual modo, cuando hablo de una proposición pensada, distingo la proposición misma del pensamiento de esa proposición" (WL 19, 77). Lo característico de las proposiciones en sí de Bolzano es que carecen de cualquier tipo de realidad fenoménica; no existen de un modo concreto en el espacio y en el tiempo (Cf. 19, 77, 137). Es esto lo que permite diferenciarlas, por una parte, de los juicios y, por otra, de las oraciones. Sin embargo, aunque propiamente hablando no existan, las proposiciones en sí son para Bolzano algo objetivo, en tanto que no dependen de un sujeto pensante; es decir, las proposiciones no tienen existencia o realidad (Wirklchkeit), pero sí tienen objetividad (Gegenständlichkeit).

Lo mismo ocurre con los elementos de las proposiciones en sí, a los que Bolzano denomina 'representaciones en sî' (Vorstellungen an sich), de las que afirma que son objetivas en el sentido de que "no requieren de un sujeto y son autosubsistentes" (WL 48, Cf. 217). En el caso de las representaciones o conceptos,

\footnotetext{
${ }^{12}$ Sobre esta cuestión, Cf. E. Morscher 1986.
} 
decir que son objetivos equivale a decir que representan algo, esto es, que hay objetos que caen (unterstehen) bajo ellos o, como hoy diríamos, que denotan algo ( Cf. WL 42, 49 y 50). Lo característico de las representaciones es, en efecto, que se refieren a algo, a objetos (WL 66), y aunque cabe distinguir también representaciones vacuas (gegestansdlos), sin embargo, en el sistema de Bolzano, las relaciones entre representaciones están sujetas a una drástica restricción: no pueden darse sino entre representaciones objetivas.

Las representaciones en sí sólo adquieren sentido, para Bolzano, en el contexto de una proposición, es decir, en una estructura de representaciones que pueda evaluarse en términos de verdad o falsedad. Bolzano, lo mismo que Frege ${ }^{13}$, confiere primacía a las proposiciones sobre los conceptos, que no son sino los constituyentes de aquéllas a los que cabe llegar mediante análisis. Las proposiciones, además de objetivas, tienen otras características entre las que destaca el hecho de ser portadoras de verdad. Verdad y falsedad son, para Bolzano, propiedades de las proposiciones (WL 24) y, del mismo modo que las representaciones se dividen en objetivas y vacuas de acuerdo con su relación con los objetos, las proposiciones se dividen, para él, en verdaderas y falsas de acuerdo con las relaciones que los objetos mantienen entre sí. El siguiente texto resume bien todas las propiedades que el lógico checo atribuye a las proposiciones en tanto que objetos lógicos centrales:

Lo que entiendo por proposición no es lo que los gramáticos denominan así, a saber, la expresión verbal, sino únicamente el sentido de esta expresión, el cual, necesariamente y siempre, no puede ser más que verdadero o falso: una proposición en st o proposición objetiva. No tengo nada que objetar a la noción de proposición en el espíritu de un ser pensante; concedo existencia a las proposiciones pensadas y a los juicios que se tienen en el espíritu del que piensa esas proposiciones y posee esos juicios. Pero a las puras proposiciones en sí, o proposiciones objetivas, las cuento entre una especie de cosas que no son en modo alguno existentes ni jamás podrán devenir tales. El que pensemos en una proposición, o juzguemos que una cosa es así o de otro modo, eso es algo real, que ha aparecido en un momento dado y desaparecerá también en un momento dado; los sig-

${ }^{13}$ Frege también confirió primacía a los juicios sobre los conceptos. Al final de su vida resumía lo distintivo de su sistema frente al de Boole y los booleanos en las siguientes palabras: «Yo no parto de conceptos y los pongo juntos para formar un juicio, sino que llego a las partes de un pensamiento analizando éste» (Cf. Frege 1979, p. 253). 
nos escritos, mediante los cuales expresamos cierta parte de las proposiciones, también son algo que pertenece a la realidad; pero las proposiciones no pertenecen a ningún tiempo ni lugar ${ }^{14}$.

En otras palabras, entre el mundo de los fenómenos y nuestros pensamientos, Bolzano postula un dominio autónomo de entidades, las representaciones en sí y las proposiciones en sí, a las que atribuye características como la atemporalidad y la inmutabilidad. Unas y otras son entidades geñerales que están más allá y son independientes de los lenguajes históricos concretos. Bolzano cree ver una anticipación de su noción de proposición en sí en un texto leibniziano, Dialogus de connexiones inter verba et res (WL 21, 27), en el que dos interlocutores discuten a propósito de si una proposición que enuncia una verdad geométrica es verdadera antes de ser demostrada y reconocida como tal por los hombres, y en el que se establece que la verdad y la falsedad son predicados del pensamiento posible (cogitatio possibilis), en tanto que distinto del pensamiento que tiene lugar en la mente del sujeto. Algunos estudiosos de la obra del checo no ven aquí sino una malinterpretación de Leibniz por su parte, ya que -alegan - el texto leibniziano no contiene en realidad una defensa de la idea de que el pensamiento es autónomo ni respecto a lo mental ni mucho menos aún respecto de su expresión lingüística, defensa que por lo demás no cuadraría del todo con la bien conocida opinión leibniziana de que el intelecto humano necesita de la ayuda de símbolos para desarrollar el pensamiento ${ }^{15}$. Mas, sea cual sea el grado de autonomía conferido por Leibniz a conceptos y pensamientos, lo que está claro es que Bolzano no pudo afirmar de modo más rotundo la independencia y autonomía de la esfera de los objetos lógicos — proposiciones y representaciones en sítanto respecto de los contenidos mentales como de sus expresiones lingüísticas. Para Bolzano, los objetos que constituyen el tema de estudio de la lógica no son objetos del mundo real, sino que habitan un tercer reino (Reich) distinto tanto del formado por los hechos del mundo físico como del constituido por los del fenómenos del mental.

Aunque los lógicos modernos se decantan más bien por la tesis de que los portadores de las propiedades y relaciones lógicas son las oraciones, entendidas

${ }^{14}$ Bolzano, Einlentung zur Grössenlehre II. La cita se toma de J. Berg 1962, pp. 47-48.

${ }^{15} \mathrm{La}$ idea de que Bolzano no interpreta adecuadamente a Leibniz en este punto ha sido defendida, entre otros, por J. Berg (Cf. Berg 1962, pp. 51-52). Para una opinión contraria a ésta, véase Mugnai 1992. 
como expresiones dotadas de un significado, la idea de que lo que constituye el objeto de la lógica es un tipo especial de objeto abstracto de índole no-lingüística ha tenido algunos otros defensores ilustres posteriores a él. $\mathrm{El}$ más importante de todos es Frege, quien también postuló la existencia de una esfera de contenidos objetivos no empíricos, con una legalidad y subsistencia propias, distinta del ámbito de las ideas o representaciones, término que, a diferencia de lo que ocurre con Bolzano, tiene siempre para Frege un sentido subjetivo ${ }^{16}$. Así, en un artículo escrito en 1880, el lógico alemán, tras establecer que el contenido del juicio que constituye el objeto de la lógica «no es el resultado de un proceso interno o el producto de un acto mental realizado por los hombres, sino que es algo objetivo", compara esta objetividad con la del sol, que explica en los siguientes términos: "¿No es acaso el sol para algunos una deidad benéfica o maléfica, para otros un disco brillante arrojado en los cielos que es lanzado del este al oeste, e incluso para otros un inmenso cuerpo esférico candente envuelto en una nube de gases incandescentes? No. A unos puede parecerles una cosa, a otros, otra ; es lo que es» (Frege 1979, p. 7).

Este tipo de postura consistente en postular un mundo de entidades matemáticas y lógicas abstractas, distinto del mundo real y fenoménico e independiente de nuestra vida mental, recibe el nombre de realismo o platonismo. De acuerdo con esta postura, de la que Bolzano es uno de los representantes más conspicuos, las proposiciones de la lógica y las matemáticas describen una realidad ideal de entidades abstractas que el lógico o el matemático se limitan a descubrir. En tanto que postura descriptivista, el platonismo se presenta como la antítesis de cualquier concepción que asuma que las proposiciones matemáticas no son sino resultado de la actividad constructiva del matemático, como es el caso de la concepción kantiana. Sin embargo, el platonismo hace causa común con el apriorismo kantiano en un aspecto: rechaza, como él, la idea de que sea posible alcanzar la verdad de este tipo de proposiciones mediante observaciones de la realidad empírica. Bolzano acepta las razones dadas al respecto por Kant, al advertir (KrV A24) que si las proposiciones matemáticas estuvieran basadas en la

${ }^{16}$ Aunque Bolzano denomina 'pensamiento' a lo que Frege llama 'representación' y que, por tanto, para el primero el pensamiento es algo subjetivo y la representación algo objetivo y para el segundo la cosa es justo al revés, la similitud de las ideas de ambos en este punto no puede ser mayor. Sin embargo, se da la circunstancia de que Frege no menciona a Bolzano ni una sola vez en toda su obra, cosa que no deja de ser extraña habida cuenta de que conocía la obra de Cantor y mantenía correspondencia con Husserl, autores ambos que no habian escatimado elogios hacia aquél. 
observación de la realidad empírica, entonces su certeza epistémica no iría más allá de la que posee cualquier proposición empírica, es decir, serían proposiciones meramente contingentes y no necesarias. No muy distintas son, en efecto, las consideraciones que llevan a Bolzano a establecer una distinción tajante entre proposiciones empíricas y proposiciones conceptuales o proposiciones que se componen de conceptos puros, distinción que en lo esencial coincide con la distinción kantiana entre proposiciones cuyo conocimiento es a posteriori y proposiciones a priori (WL 133, n.). Sin embargo, al entender que el establecimiento de la verdad de las proposiciones conceptuales ha de efectuarse de manera racional, es decir, sin recurso a la intuición, Bolzano se aparta de la teoría kantiana de lo a priori para proponer una explicación alternativa, explicación que no podremos entender sin antes analizar su noción de analiticidad que, como veremos, es un tanto distinta de la de Kant.

\section{Variación de conceptos y analiticidad}

Pese a su familiaridad con las ideas de Leibniz y a la innegable influencia que éste ejerció sobre muchos de los aspectos de su pensamiento, Bolzano no acudió, sin embargo, a la noción leibniziana de mundo posible a la hora de definir la verdad lógica, tal vez por ser consciente de la circularidad a que está abocada cualquier definición que identifique la verdad lógica con la proposición verdadera en todos los mundos posibles si por tal no da en entenderse otra cosa que mundo lógicamente posible, esto es, un mundo que no viola las leyes de la lógica. El lógico checo consideró más fructífera a estos efectos la noción de analiticidad, que tomó directamente de Kant, si bien no se atuvo estrictamente al sentido que éste había conferido al término.

Como es bien sabido, reelaborando Ja distinción leibniziana entre verdades de razón y verdades de hecho, Kant introdujo la doble distinción entre juicios a priori y juicios a posteriori, de un lado, y juicios analíticos y sintéticos, de otro. Entiende Kant por juicios analíticos aquel tipo de juicios «que no añaden nada al concepto del sujeto mediante el predicado" (KrV B 10-11), sino que se limitan a descomponerlo en sus conceptos componentes. Así por ejemplo, dado que ser cuadrado aúna las dos propiedades de ser equilátero y ser rectángulo, el predicado del juicio expresado por la oración «Todos los cuadrados son rectángulos» no haría otra cosa que 'analizar' lo que se halla contenido en el sujeto, de suerte que todo juicio analítico podría expresarse como una simple redundancia (en este caso como "Todo lo que es equilátero y rectángulo es rectángulo»). Negar esta re- 
dundancia sería afirmar una contradictio in adjecto, ya que no sería sino afirmar que hay cosas que tienen y no tienen una cierta propiedad (por ejemplo, la de ser y no ser rectángulo). De ahí que Kant, termine estableciendo que el principio común a todo juicio analítico sea la ley de contradicción (KrV B190).

Sin dejar de reconocerle a Kant el mérito de haber sido el primero en aplicar fructíferamente esta distinción (WL 148 n.4), Bolzano cree, sin embargo, que los términos 'analítico' y 'sintético' están necesitados de una caracterización más precisa que la suya. Hay una doble razón, a juicio del lógico checo, para considerar inadecuada la noción kantiana de analiticidad: la primera es que, conforme al criterio kantiano, en la categoría de los juicios analíticos no tendrían cabida una clase importante de verdades lógicas, como por ejemplo, el principio del tercio excluso, cuya analiticidad nadie discutiría, y la segunda, que, conforme a tal criterio, serían en cambio analíticos juicios que en modo alguno lo son, como ocurre, por ejemplo, con «El padre de Alejandro, rey de Macedonia, fue rey de Macedonia", que no es analítico a pesar de que el predicado esté contenido en el sujeto (WL 148). Ahora bien, a Bolzano es precisamente esta noción de analiticidad la que más le interesa de la distinción kantiana; de ahí que considere un reto el tratar de llegar a una explicación más exacta y precisa que la ofrecida por el filósofo alemán. Para ello, acude a un par de nociones previas, la de sustitución de representaciones y la de validez, que no habrá más remedio que analizar aunque sólo sea someramente antes de ver cuál es en concreto su concepto de analítico.

El método de la variación o sustitución de representaciones en una proposición, además de constituir la base misma a partir de la cual se desarrolla gran parte de la lógica proposicional de Bolzano, es el instrumento del que se vale el filósofo checo para analizar las propiedades lógicas de las proposiciones, como es el caso de la analiticidad. Bolzano observó que, igual que una función en matemáticas puede adoptar diferentes valores cuando se sustituyen las variables de sus argumentos, así también una proposición puede cambiar su valor de verdad si se cambian ciertos componentes de la misma. Así, la proposición verdadera

Cayo es mortal

puede convertirse en la falsedad

Cayo es omnisciente 
si variamos la representación o idea correspondiente al predicado. Pero también podríamos tomar como representación variable de la misma a 'Cayo'. La diferencia es que, en este caso, cada sustitución adecuada, entendiendo por tal aquella cuyo resultado no es vacío, genera siempre una proposición verdadera: 'Tito es mortal', 'Sempronio es mortal', etc.

Una forma proposicional (Sätzform), o la proposición correspondiente en la que ciertos componentes se consideran variables, puede generar toda una clase de proposiciones siempre y cuando las representaciones variables se sustituyan por representaciones apropiadas, cosa que, para él, quiere decir que cumplan con el requisito de que la representación-sujeto de las mismas sea objetiva o no vacua (WL 127, 146) ${ }^{17}$. A la proposición que es resultado de la sustitución la denomina Bolzano 'variante' de la proposición (o forma proposicional) dada. Conviene indicar que, pese a esta forma de hablar, Bolzano no posee en realidad la noción de función proposicional en el sentido plenamente moderno de la expresión. Además de no utilizar siempre variables para indicar los elementos sujetos a variación dentro de una proposición ${ }^{18}$, las variables no están nunca por objetos, como en la lógica actual, sino que están por representaciones que asumen el papel bien de sujeto, bien de predicado, ya que el análisis que Bolzano hace de la proposición sigue siendo el análisis tradicional en términos del patrón sujeto/predicado.

Una de las debilidades más grandes del sistema lógico de Bolzano es su estrecha vinculación al lenguaje natural y la ausencia de un lenguaje formal en el que plantear de un modo más adecuado muchos de los problemas suscitados. El filósofo checo parte del supuesto de que existe una correlación exacta entre ciertas estructuras del lenguaje natural y ciertos fenómenos lógico-semánticos, sirviéndose para la exposición de su sistema de un lenguaje que podríamos considerar semiformalizado, ya que no es otro que el tenguaje alemán común al que se aña-

${ }^{17}$ La exigencia de objetividad en el uso de las representaciones restringe severamente el sistema de Bolzano. Un efecto importante de esta exigencia es el rechazo, por parte de Bolzano, de las demostraciones indirectas o por reducción al absurdo, que para él sólo sirven para la presentación de resultados, pero no tienen valor de demostración.

${ }^{18}$ Por lo general, Bolzano sólo utiliza letras variables cuando opera con formas proposicionales propiamente dichas, esto es, con formas en las que todos los componentes no-lógicos se consideran sujetos a variación. Cuando habla de proposiciones que contienen algún elemento no sujeto a variación, lo que hace es escribir la proposición completa e indicar a continuación cuál o cuáles términos se consideran variables en ella. 
den algunos términos técnicos como elementos primitivos del mismo. Un primer fruto de esta postura es la postulación de una forma única para todas las proposiciones, que son tomadas como ejemplificaciones del patrón sujeto-predicativo, siendo la única novedad respecto de la tradición que en lugar de optar por 'ser' como la cópula encargada de vertebrar la proposición, él prefiere hablar de 'poseer' (haben). De acuerdo con esta suposición, 'A posee b' constituye la forma canónica de todas las proposiciones (en donde ' $A$ ' es la representación-sujeto y se refiere a un objeto; ' $\mathrm{b}$ ' es la propiedad o representación-predicado que se atribuye a ese objeto y 'posee' es la cópula (WL 81, 126-127)).

Esta decisión de contemplar todas las proposiciones como ejemplificaciones de este único modelo hace que se vea abocado a expresar ciertas nociones generalmente consideradas como formales mediante símbolos que en sus formas reductivas desempeñan el mismo papel que los reservados para la expresión de conceptos materiales. Así, por ejemplo, la negación, que es tomada como formando parte del predicado, se expresa en su sistema como 'A posee falta de b' ( $A$ bat der Mangel an b). Esto es, una proposición como 'Alcibíades no es sabio' se reduce, en el sistema de Bolzano, a 'Alcibíades posee falta de sabiduría'; una proposición particular, 'Algún A es B', se convierte, de acuerdo con su análisis, en 'La representación de un A que tiene b tiene objetividad' (es decir, no es vacua), 'Ningún A es B', en 'La representación de un A que tiene b tiene vacuidad', etc. (Cf. WL 137, 138). Aunque Bolzano mostró no poco ingenio a la hora de hacer encajar diversos tipos de proposiciones dentro de esta única horma, su apego a la tradición en este punto y su actitud negativa hacia el desarrollo de una escritura simbólica en la que poder expresar con mayor precisión las relaciones lógicas y su consiguiente opción de servirse para este fin del lenguaje natural constituyen, sin duda, el principal escollo de toda su teoría lógica. De hecho, fueron un obstáculo que le impidió sacar mayor provecho de algunas de sus originales ideas que no reaparecerían en el ámbito de la lógica sino bastante tiempo después.

El método de la variación de representaciones en una proposición constituye, pese a sus insuficiencias, la base a partir de la cual se desarrolla gran parte de la lógica proposicional de Bolzano. Las propiedades lógicas más importantes de las proposiciones, lo mismo que las relaciones entre ellas, sólo se ponen de manifiesto, dice Bolzano, cuando consideramos ciertas representaciones en sí contenidas en ellas como algo variable y observamos al mismo tiempo el comportamiento de las nuevas representaciones, obtenidas por variación, en lo que atañe a la verdad y la falsedad (WL 154). Dos de las nociones que Bolzano va a caracterizar con ayuda de este recurso son la de validez universal y la de analiticidad. 
El argumento forjado por Bolzano para introducir la noción de la validez general (Allgemeingültigkeit) discurre del modo siguiente: la mayoría de las proposiciones están constituidas de tal suerte que las proposiciones que se pueden generar a partir de ellas por sustitución de sus elementos constitutivos no son ni todas verdaderas ni todas falsas, sino sólo algunas de ellas verdaderas y algunas falsas. Pero hay proposiciones que son de tal naturaleza que todas las que pueden derivarse a partir de ellas por sustitución de algún elemento constitutivo concreto son verdaderas: de ellas diremos que son universalmente válidas por respecto a la representación cuya sustitución ha sido tomada en consideración; hay en cambio otras proposiciones capaces de generar por sustitución de alguno de sus componentes una clase de proposiciones falsas; en este caso diremos de la proposición originaria que es inválida con respecto al elemento o elementos constitutivos cuya variación ha sido tomada en consideración. Así, por ejemplo, la proposición 'Cayo es mortal' es universalmente válida relativamente a la representación variable 'Cayo', porque cada sustitución apropiada genera siempre una proposición verdadera; en cambio, la proposición 'Cayo es omnisciente' sería inválida relativamente a la misma representación, 'Cayo', ya que todas las sustituciones de la misma generan una proposición falsa.

Es de este modo como Bolzano llega por fin a la noción de analiticidad, que en su sistema no es sino una generalización de esta noción de validez universal. Validez e invalidez son en Bolzano, como acabamos de ver, nociones relativas, toda vez que dependen de las representaciones consideradas. Una proposición es analítica si entre las representaciones que la conforman hay al menos una respecto de la cual es universalmente válida (analíticamente verdadera) o universalmente inválida (analíticamente falsa), siendo sintéticas aquellas proposiciones que no contienen ninguna representación que pueda ser sometida a variación arbitraria sin que ello afecte a su valor de verdad (WL 148. 1). Bolzano ilustra esta nueva noción con dos ejemplos: 'un hombre moralmente malo no merece ningún respeto' es, para él, un ejemplo de proposición analíticamente verdadera, dado que contiene una representación variable, 'hombre', cuya sustitución sólo dará lugar a proposiciones verdaderas; 'Un hombre moralmente malo goza de felicidad eterna' es, para él, igualmente analítica, aunque falsa, porque contiene la variable 'hombre' relativamente a la cual todas las variantes son falsas (Ibid.).

Bolzano admite abiertamente que la noción de proposición analítica así definida es bastante más amplia que la de Kant. Efectivamente, la suya es una definición tal que resulta aplicable no sólo a las proposiciones verdaderas, como la de 
aquél, sino también a las falsas. Por otro lado, la caracterización de Bolzano parte de la idea de que la analiticidad es un atributo de las proposiciones, y no de las oraciones que las expresan, basándose en la presuposición de que las proposiciones han de contener elementos constitutivos en absoluta correspondencia con todos y cada uno de los elementos de la oración ${ }^{19}$. Pero se trata de una definición que resulta demasiado amplia para ser útil ${ }^{20}$, y que carecería de interés si no fuera porque constituye un paso necesario para su definición de la noción más estricta de proposición lógicamente analítica, de la que nos ocupamos a continuación.

\section{Verdad lógica y consecuencia lógica}

El método de la variación o sustitución no sólo es importante en la obra de Bolzano por constituir la base para la definición de las nociones de validez y analiticidad. Debe sobre todo su interés al hecho de que es el que confiere a la lógica su carácter formal y posibilita el que ésta trate no de proposiciones concretas (einzelne Sätze) sino de lo que Bolzano denomina 'formas generales de proposiciones' (allgemeine Formen der Sätze), entendiendo por tales precisamente aquellas expresiones obtenidas a partir de proposiciones concretas por el procedimiento de declarar variables o susceptibles de sustitución ciertas representaciones no lógicas (WL 12, Cf. 48, 81). Esta noción de forma cobra especial relieve en la obra del lógico checo debido sobre todo a que constituye la base de su explicación de dos conceptos fundamentales de la lógica: el de verdad y el de consecuencia lógica.

Después de introducir una noción de analiticidad que, como antes hemos visto, resultaba excesivamente amplia para ser útil, Bolzano pasa a definir lo que

${ }^{19}$ Ya hemos señalado que esta suposición resulta un tanto ingenua. De hecho, aunque para Bolzano la analiticidad es un atributo de las proposiciones, en más de una ocasión Bolzano se refiere claramente a oraciones y no a proposiciones. Una de las dificultades con que tropieza este apartado de la teoría de Bolzano es el del establecimiento de un criterio de identidad para proposiciones, dado que, por ejemplo, una misma proposición puede ser expresada por diferentes oraciones (WL 127).

${ }^{20}$ En una de las primeras exposiciones del sistema de Bolzano, W. y M. Kneale le acusaron de haber ofrecido una noción tan sumamente amplia de analiticidad que «una proposición podría ser analítica por obra y gracia de una ley natural y hasta de un simple accidenten. Esto es lo que sucedería, en su opinión, con una oración como "Kant no fue un filósofo del siglo dieciocho que muriese el día del aniversario de su nacimiento", pues si fuese el caso de que ningún filósofo del dieciocho hubiese muerto el día del aniversario de su nacimiento, todas las oraciones resultantes de sustituir Kant por otros nombres resultarían verdaderas (Cf. W y M. Kneale 1972, p. 339). 
él denomina analiticidad lógica o analiticidad en sentido estricto, que no es sino nuestra noción de verdad lógica. En efecto, en la misma sección del libro II de Wissenschaftslehre, después de decirnos que una proposición es analítica en un sentido amplio si es válida o inválida con respecto a un constituyente dado y de poner como ejemplos de la misma las dos proposiciones citadas al final del punto anterior (148.1), Bolzano ofrece otra serie de ejemplos — $\mathrm{A}$ es $\mathrm{A}$, A que es B es A, A que es B es B, Todo objeto es B o no B, etc.) (WL 148.2) - que a todas luces son de distinta índole que los anteriores y añade a continuación:

Los ejemplos de proposiciones analíticas que acabo de ofrecer se distinguen de los incluidos en (1) por el hecho de que para juzgar la naturaleza analítica de los mismos no se necesita otra cosa que conocimiento lógico, dado que los conceptos que constituyen la parte invariante de esas proposiciones pertenecen todos a la lógica, en tanto que el juzgar la verdad o falsedad de proposiciones como las de (1) requiere un tipo de conocimiento muy distinto, toda vez que intervienen en ella conceptos ajenos a la lógica. Desde luego, esta distinción es más bien fluctuante [hat sein Schwankendes], por cuanto que el dominio total de los conceptos pertenecientes a la lógica no está delimitado de manera tan precisa [so scharf begrenzt] que no pueda surgir nunca alguna controversia sobre el tema. En ocasiones podría ser útil prestar atención a esta distinción, y así podríamos denominar a proposiciones como las de (2) lógicamente analíticas o analíticas en sentido estricto y a las de (1) analíticas en sentido amplio (WL 148.3).

Sería difícil exagerar la importancia de este texto, del que se ha dicho que constituye el acta de nacimiento del concepto de verdad lógica ${ }^{21}$. Hay razones para ello: Bolzano está aquí afirmando que hay un tipo de proposiciones cuyas variantes relativamente a todos los elementos constitutivos que no sean términos lógicos son todas ellas verdaderas y que lo son, por tanto, tan sólo en virtud de la forma lógica que poseen. Bolzano llega a insinuar que nadie ha reparado en esta característica de este tipo de proposiciones cuando, tras pasar revista, según es su costumbre, a una serie de tesis mantenidas por otros autores acerca de la analiticidad, concluye:

${ }^{21}$ Bar-Hillel, uno de los primeros estudiosos de la obra de Bolzano, considera que este párrafo encierra "uno de los logros lógicos más interesantes e ingeniosos de todos los tiempos" (BarHillel 1950, p.101). Cf. también Sebestik 1992, p. 281. 
En general, me parece que ninguna de estas definiciones pone demasiado énfasis en lo que hace realmente importantes a este tipo de juicios [analíticos]. A mi modo de ver esto estriba en el hecho de que su verdad o falsedad no depende de las representaciones concretas de las que están constituidos, sino que se mantiene idéntica sean cualesquiera que sean los cambios que se introduzcan en algunos de ellos, presuponiendo tan sólo que el carácter objetivo de la proposición no resulte destruido (WL 148.4).

Ya hemos visto cómo un presupuesto subyacente a la lógica bolzaniana es el de que todas las proposiciones en sí mismas han de ser objetivas, esto es, han de cumplir el requisito de que su representación-sujeto sea objetiva y no vacua (WL 146). Dejando ahora de lado la limitación que para el sistema supone semejante presupuesto, lo verdaderamente sobresaliente en este texto es que en él se halla implícito un claro reconocimiento de que hay ciertas características que corresponden a las proposiciones en virtud de su forma, es decir, que corresponden a esquemas proposicionales más bien que a las proposiciones que puedan ejemplificarlos. De hecho, la definición de Bolzano anticipa claramente la ofrecida por Tarski un siglo después. Como es sabido, Tarski definió (en Tarski 1936) las oraciones analíticas como aquellas que son verdaderas en todo modelo, siendo ésta posiblemente la primera definición dada de oración analítica en términos semánticos. Tarski añade a continuación un comentario que me parece interesante reproducir porque creo que pone de manifiesto la necesidad de revisar la historia de la analiticidad que nos ha legado el positivismo lógico. Es el siguiente: «Según muchos lógicos, este concepto [analítico] ha de considerarse como el correlato formal del concepto de tautologia (i.e., de enunciado que 'no dice nada acerca de la realidad'), concepto que a mí personalmente me parece más bien vago, pero que ha sido de fundamental importancia para las discusiones de L. Wittgenstein y de todo el Círculo de Viena" (Tarski 1983, págs. 419-20).

Bolzano no sólo hace de la forma lógica el centro de su explicación de la analiticidad lógica sino que también la convierte en la base de su caracterización de la relación de deducibilidad o consecuencia lógica $(\text { Ableitbarkeit })^{22}$, caracterización que de nuevo anticipa la ofrecida por Tarski en términos mucho más preci-

${ }^{22}$ En realidad, aunque lo normal es traducir este vocablo por 'deducibilidad', la relación así nombrada por Bolzano no se corresponde con la relación sintáctica de deducibilidad o derivabilidad, sino con la de consecuencia lógica. No hay que confundir esta relación con la llamada por 
sos en el ya citado artículo de $1936^{23}$. Bolzano define esta noción en los siguientes términos: «Digo que las proposiciones $\mathrm{M}, \mathrm{N}, \mathrm{O}$... serían derivables [Abletbar] de las proposiciones A, B, C, D..., respecto de los elementos constitutivos $i, j, \ldots$ si todo conjunto de representaciones que hace verdaderas a $A, B, C, D$...cuando se las pone en el lugar de $i, j$..., también hace verdaderas a $M, N$, O... Para variar, a veces también diré que las proposiciones $\mathrm{M}, \mathrm{N}, \mathrm{O}$...se siguen de o pueden inferirse o concluirse del conjunto de proposiciones A, B, C, D...» (WL 155).

Bolzano creyó ver un claro precedente de esta noción en el modo en que Aristóteles entendió la necesidad de las implicaciones silogísticas correctas, ya que, en su opinión, cuando Aristóteles se sirvió de la expresión 'se sigue de necesidad' para describir la relación que media entre premisas y conclusión de un silogismo válido, lo que quiso decir en realidad es que todo argumento de la forma especificada conduce a una conclusión verdadera sólo con tal de que las premisas lo sean (WL 223). No es el momento de discutir el fundamento de esta creencia ni de analizar si Aristóteles entendió exactamente en estos términos esta noción, cosa que resulta discutible. Limitándonos a la consideración de la definición bolzaniana, lo primero que habría que destacar es que se trata de una definición en la que la relación de deducibilidad (hoy conocida como 'consecuencia lógica') es entendida como una relación formal entre proposiciones, pues decir que la deducibilidad es una relación entre proposiciones en que se consideran variables todos los elementos constitutivos excepto las partículas lógicas equivale a decir que es una relación que no se basa en otra cosa que la pura forma de las proposiciones (die blosse Form der Sätze $)$. Así lo subraya Bolzano cuando afirma que para reconocer que de las premisas 'Cayo es un hombre' y 'Todos los hombres tienen un alma inmortal' se sigue como conclusión 'Cayo tiene un alma inmortal', "no es preciso nada más que el conocimiento de la verdad universal siguiente: de dos proposiciones de la forma $\mathrm{A}$ es $\mathrm{B}$ y $\mathrm{B}$ es $\mathrm{C}$ se puede deducir una tercera de la forma $\mathrm{A}$ es C. Y esto puede verse sin saber nada de la naturaleza de los seres humanos, de lo que quiere decir morir, y demás" (WL 223).

Bolzano 'consecuencia fundamental' ( $A b f o l g e$ ), en la que pueden distinguirse como componentes un conjunto de proposiciones, el fundamento de cierta proposición y la consecuencia ( $F o$ lge) de las mismas. Algunos autores ( $\mathrm{J}$. Berg, por ejemplo) traducen este término por 'entailment' (implicación), pero lo normal es hablar de 'consecuencia fundamental' y reservar el nombre de deducibilidad para Ableitbarkeit.

${ }^{23} C f .1983$, p. 417 . En una nota de esa misma página, Tarski dice haberse enterado por un artículo de $\mathrm{H}$. Scholz de la similitud de su definición con la de Bolzano. 
El problema de convertir a la forma lógica en la noción central tanto de la definición de verdad lógica como de la caracterización de la relación de consecuencia estriba en que la clarificación de esta noción se basa en la posibilidad de establecer una clara línea de demarcación entre los términos que forman parte del vocabulario lógico y los puramente descriptivos, entre la clase de los términos que los medievales llamaban sincategoremáticos y la de los categoremáticos. Tarski se declara claramente escéptico respecto de que exista esta posibilidad al afirmar:

En la base de toda nuestra construcción se halla la división de los términos del lenguaje en lógicos y extralógicos. Tal división no es desde luego una división arbitraria....Pero, por otro lado, no sé de fundamento alguno que permita trazar una clara línea divisoria entre los dos grupos de términos. No parece imposible incluir entre los términos lógicos algunos de los usualmente considerados por los lógicos como extralógicos sin que de ello se desprendan consecuencias que están en claro contraste con la práctica ordinaria. Y en el caso límite de semejante posibilidad, todos los términos de nuestro lenguaje podrían considerarse como lógicos (Tarski, 1983, págs. 418-19).

El hecho de que Tarski manifieste sus dudas respecto de esta posibilidad en términos tan similares a los empleados por Bolzano ( $C f$. WL 148, final del texto reproducido más arriba), junto con otras similitudes ya señaladas, parece indicar que el pensamiento del filósofo checo no era del todo ajeno a la escuela de Varsovia en donde tuvo lugar la formación de Tarski y el desarrollo de su actividad investigadora hasta que la barbarie nazi le obligó a emigrar a Estados Unidos. Aunque no es mucho lo que sabemos acerca de la propagación de las ideas de Bolzano, lo que sí parece claro es que K. Twardowski, el fundador de la escuela polaca, estaba familiarizado con ellas por haber hecho su doctorado bajo la supervisión de $\mathrm{R}$. Zimmermann, discípulo de aquel y encargado de su legado, y que es sumamente probable que tales ideas llegaran hasta Tarski a través de ciertos discípulos de aquél, especialmente Lukasiewicz y Lesniewski ${ }^{24}$. Y es que, en la historia de la ciencia, no es infrecuente que aparezcan ideas y desarrollos que en su momento son ignorados por las concepciones dominantes de

${ }^{24} \mathrm{La}$ influencia de Bolzano sobre Twardowski especialmente en lo que se refiere a la doctrina de que la verdad es absoluta y de que la presencia de expresiones indéxicas no entraña la relarividad de la misma es innegable, toda vez que, el segundo se sirve incluso de alguno de los ejemplos puestos por el primero en defensa de la misma. Cf. Simons 1992, p. 15. 
los temas con los que están relacionados y que reaparecen con todo su vigor años e incluso siglos más tarde. Esto es lo que ha sucedido no ya con la noción de consecuencia de Bolzano sino incluso con lo que podríamos considerar el logro principal de su obra: la separación de las proposiciones y representaciones objetivas del proceso psicológico que tiene lugar cuando pensamos y la estipulación de una esfera de contenidos objetivos, con una legalidad y subsistencia propias, como esfera de la lógica.

Resumiendo: Bolzano pensaba que, si bien muchas de las distinciones introducidas por Kant son útiles y correctas, no todas lo son, y que, de hecho, algunos de los presupuestos más importantes de la filosofía kantiana (como, por ejemplo, su idea de una intuición pura) descansan en un deficiente análisis conceptual. Éste sería en concreto, a su juicio, el origen de las limitaciones de la concepción kantiana en lo referente a la filosoffa de la matemática y a la lógica. En lo que respecta al papel de esta última en relación con las matemáticas, las posturas de ambos autores no puede estar, en efecto, más alejadas: si para Bolzano la lógica constituye el fundamento mismo de la verdad matemática y de su metodología, para Kant resulta enteramente ajena al mismo; y si para aquél tales verdades son algo objetivo y autónomo, para éste consisten más bien en el resultado de la actividad de un sujeto, de la que en consecuencia dependen. En su obra se encuentra, en efecto, el primer intento de explicación del ámbito de lo a priori desde un punto de vista puramente lógico, es decir, sin recurso a la intuición, así como la primera crítica a la concepción psicologista de la lógica. Pero Bolzano no sólo contribuyó al desarrollo de la lógica a través de su oposición al psicologismo y de su reivindicación de un estatuto autónomo y objetivo para esta disciplina. Lo hizo también con la aportación de sus análisis pormenorizados de algunos conceptos cardinales de la lógica, como los de verdad lógica y consecuencia lógica. Fue así uno de los primeros lógicos en mostrar por la vía de los hechos el error en el que estaba el gran filósofo alemán — su gran oponente filosófico- al pensar que la lógica debía considerarse algo ya concluido y perfecto.

\section{Bibliografía}

BAR-HILLEL, Y.: 1950, “Bolzano's Definition of Analytic Propositions", Theoria 16, 91-117. BERG, J.: 1962, Bolzano's Logic, Stokholm, Almqvist \& Wiksell.

BerGmann, H.: 1909, Das philosophische werk Bernard Bolzanos (reimp. New York, Hildesheim, 1970). 
BolzANO, B.: 1810, Beiträge zu einer begründeteren Darstellung der Mathematik, en L. Novy, B. Bolzano 1781-1848. Early Mathematical Work, Praga, CSAS, 1981.

BolZANO, B.: 1837, Wissenschaftslehre. Versuch einer ausfübrlichen und gröstentheils neuen Darstellung der Logik mit Steter Rücksicht auf deren Bisherige Bearbeiter, en Gesamtausgabe, Reihe I, 11-14 Schriften, Stuttgart-Bad-Cannstatt, Friedrich Fronmann Verlag, 1985-2000.

Boswell, T.: 1988, "On the textual authenticity of Kant's logic", History and Philosophy of Logic 9, 193-203.

FrEGE, G.: 1979, Posthumous Writings, edit. H. Hermes, Chicago, Chicago University Press.

GeORGE, R.: 1997, "Psychologism in logic: Bacon to Bolzano», Philosophy and Rhetoric 30, 213-241.

Haller, R.: 1992, "Bolzano and Austrian Philosophy", en VV.AA. (1992): 191-206.

HUSSERL E.: Logische Untersuchungen, Freiburg, Niemayer, 1968.

KANT I.: $1 .^{a}$ edición: 1781; $2 .^{a}$ edición: 1787, Kritik der reinen Vernunft, en $A k$ Kant's gesammelte Schriften, Berlín Walter de Gruyter, 1902-, vol. 4.

KANT, I.: Jäsche Logic, en Ak, vol. 9. Trad.al inglés: Lectures on Logic, tr. Michael Young, Cambridge, Cambridge University Press, 1992.

KusCH, M.: 1995, Psychologism. A Case Study in the Sociology of Philosophical Knoledge, London, Routledge.

KNEALE, W. y M.: 1972, El desarrallo de la lógica, Madrid, Tecnos.

MORSCHER, E.: 1973, Das logishe an-sich bei Bernard Bolzano, Salzburg, München.

MORSCHER, E.: 1986, "Propositions and states of Affairs in Austrian Philosophy before Wittgenstein", en J.C. Nyíri (1986): 75-85.

MuGNAI, M.: 1992, “Leibniz and Bolzano on the 'Realm of Truths', en VV.AA. (1992): 207-220.

Mulligan, K. 1986, «Exactness, Description and Variation : How Austrian Analytical Phylosophy was done», en J.C. Nyíri (1986): 86-98.

NYfRI, J.C.: 1986, Von Bolzano zu Wittgenstein, Viena, Hölder-Pichler-Tempsky.

SEBESTIK, J.:1992, Logique et mathematique chez Bernard Bolzano,Paris, Librairie Philosophique J. Vrim.

Simons, P.: 1992, Philosophy and Logic in Central Europe from Bolzano to Tarski, Dordrechr- Boston, Kluwer Academic Publishers. 
TARSKI, A.: 1936, «On the concept of Logical Consequence», en Tarski, A., Logic, Semantics, Methamatematics, Indianapolis, Hackett, 1883: 409-420.

VV.AA. 1992, Bolzano's Wissenschaftslehre 1837-1987, International Workshop (Firenze 1987), Firenze.

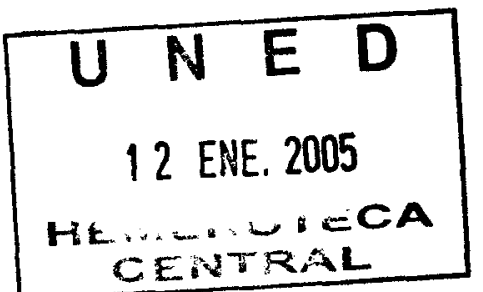

